\title{
Latitude and HLA-DRB1*04:05 independently influence disease severity in Japanese multiple sclerosis: a cross-sectional study
}

Yuri Nakamura', Takuya Matsushita', Shinya Sato', Masaaki Niino², Toshiyuki Fukazawa ${ }^{3}$, Satoshi Yoshimura', Shin Hisahara ${ }^{4}$, Noriko Isobe', Shun Shimohama ${ }^{4}$, Mitsuru Watanabe ${ }^{1}$, Kazuto Yoshida ${ }^{5}$, Hideki Houzen ${ }^{6}$, Yusei Miyazaki ${ }^{2,7}$, Ryo Yamasaki ${ }^{1}$, Seiji Kikuchi ${ }^{7}$, Jun-ichi Kira ${ }^{1 *}$ and the Japan Multiple Sclerosis Genetics Consortium

\begin{abstract}
Background: Higher latitude and human leukocyte antigen (HLA)-DRB1*04:05 increase susceptibility to multiple sclerosis (MS) in the Japanese population, but their effects on disease severity are unknown. We aimed to clarify the effects of latitude and the HLA-DRB1 and HLA-DPB1 genes on disease severity in Japanese patients with MS.

Methods: We enrolled 247 MS patients and 159 healthy controls (HCs) from the northernmost main island of

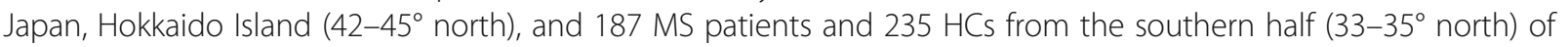
the Japanese archipelago (33-45 north). We genotyped HLA-DRB1 and HLA-DPB1 alleles, compared demographic features, and analyzed factors contributing to differences in clinical and laboratory findings between MS patients from southern and northern Japan. The Multiple Sclerosis Severity Score (MSSS), which adjusts the Kurtzke's Expanded Disability Status Scale score according to disease duration, was used to estimate disease severity.
\end{abstract}

Results: The HLA-DRB1*04:05 and DRB1*15:01 alleles conferred susceptibility to MS in our Japanese population ( $p^{\text {corr }}=0.0004$ and $p^{\text {corr }}=0.0019$, respectively). Southern patients had higher MSSS scores than northern patients $(p=0.003)$. Northern patients had higher frequencies of brain lesions meeting the Barkhof criteria (Barkhof brain lesions) and cerebrospinal fluid (CSF) IgG abnormalities than southern patients ( $p=0.0012$ and $p<0.0001$, respectively). DRB1*04:05-positive MS patients had lower MSSS scores and lower frequencies of Barkhof brain lesions and CSF IgG abnormalities than DRB1*04:05-negative MS patients $(p=0.0415, p=0.0026$, and $p<0.0001$, respectively). Multivariate analyses revealed that latitude and DRB1*04:05 were independently associated with the lowest quartile of MSSS and that latitude was positively associated with Barkhof brain lesions and CSF IgG abnormalities. DRB1*04:05 was negatively associated with these parameters. MSSS was decreased by 0.57 per DRB ${ }^{*} 04: 05$ allele $(p=0.0198)$.

Conclusions: Living at a higher latitude and carrying the $D R B 1^{*} 04: 05$ allele independently lessens MS symptom severity as defined by MSSS. However, these factors influence the frequency of Barkhof brain lesions and CSF IgG abnormalities in opposite ways; higher latitude increases the frequency of Barkhof brain lesions and CSF IgG abnormalities, whereas DRB 1*04:05 decreases them.

(Continued on next page)

\footnotetext{
* Correspondence: kira@neuro.med.kyushu-u.ac.jp

${ }^{1}$ Department of Neurology, Neurological Institute, Graduate School of

Medical Sciences, Kyushu University, 3-1-1 Maidashi, Higashi-ku, Fukuoka

812-8582, Japan

Full list of author information is available at the end of the article
} 
(Continued from previous page)

Keywords: Multiple sclerosis, HLA, Latitude, Magnetic resonance imaging, Oligoclonal lgG bands

Abbreviations: $\mathrm{Cl}$, Confidence interval; CSF, Cerebrospinal fluid; DMDs, Disease-modifying drugs; EDSS, Expanded Disability Status Scale; HCs, Healthy controls; HLA, Human leukocyte antigen; MS, Multiple sclerosis; MSSS, Multiple Sclerosis Severity Score; NA, Not applicable; NS, Not significant; OB, Oligoclonal IgG bands; OR, Odds ratio;

SPMS, Secondary progressive multiple sclerosis

\section{Background}

Multiple sclerosis (MS) is a chronic disease of the central nervous system characterized by inflammation, demyelination, and axonal injury [1]. MS susceptibility and phenotypes result from complex interactions between multiple genetic and environmental factors [2]. It is well established that the class II subregion of the human leukocyte antigen $(H L A)$ gene on chromosome 6p21 plays the most important role in determining MS susceptibility [3]. The DR15 haplotype (DRB1*15:01$D Q A 1 * 01: 02-D Q B 1 * 06: 02)$ is strongly associated with MS, and the effect is mainly driven by $H L A-D R B 1 * 15: 01$ in people of northern European descent $[4,5]$. Although $D R B 1$ alleles clearly influence MS susceptibility, it is not known how $H L A$ variation influences the clinical features of MS, especially in Asian populations.

We previously reported that the HLA-DRB1*04:05 allele is the strongest genetic risk factor for MS in the southern Japanese population. The clinical phenotypes of patients with the HLA-DRB1*04:05 allele include a milder clinical course, younger age at disease onset, slower disease progression, fewer brain lesions, and rare cerebrospinal fluid (CSF) IgG abnormalities compared with patients lacking the HLA-DRB1*04:05 allele [6]. It is widely known that the risk for MS increases with distance from the equator [7], suggesting that environmental risk factors related to latitude, such as decreased sunlight exposure and vitamin D levels, are involved [8]. Additionally, despite genetic homogeneity, there are phenotypic differences between northern and southern Japanese patients with MS. In the fourth nationwide survey in Japan, brain magnetic resonance imaging (MRI) findings fulfilling the Barkhof criteria [9] were significantly more common in northern patients than in southern patients and more frequent in northernborn northern residents than northern-born southern residents [10]. Recent studies in Western countries have revealed an association between latitude and the presence of oligoclonal IgG bands (OB) or increased intrathecal production of IgG $[11,12]$. We have also reported that latitude independently affects the emergence of CSF IgG abnormalities in Japanese patients with MS [13].

These observations suggest that latitude may influence the clinical phenotype of MS. However, the relationship between latitude and disease severity still remains unclear. Thus, the aim of this study was to clarify the influence of latitude and $H L A-D R B 1$ on disease severity in Japanese patients with MS.

\section{Methods \\ Participants}

Patients with MS, agreeing to participate in this study, were recruited from nine institutes in Japan. MS was diagnosed using the 2010 revised McDonald criteria [14]. Patients with neuromyelitis optica [15], neuromyelitis optica spectrum disorders [16], or longitudinally extensive spinal cord lesions extending over three or more vertebral segments were excluded. Patients with primary progressive MS (PPMS) were also excluded because of the low prevalence of PPMS in the Japanese population [10], as well as the association between PPMS and distinct $H L A$ class II alleles from relapse-onset MS [17, 18]. Participants were recruited from the northern and southern parts of the Japanese archipelago, which spans $33-45^{\circ}$ north (Additional file 1: Figure S1). Patients recruited from Hokkaido Island, the northernmost main island of Japan ( $42-45^{\circ}$ north), were classified as the northern group, and those recruited from the southern half of the Japanese archipelago (33-35 $5^{\circ}$ north) were classed as the southern group. Patients residing on Hokkaido were chosen for the northern group because most people living on Hokkaido are descendants of immigrants who came from the other main islands 100150 years ago, during the Meiji era, and thus share similar genetic makeup with modern inhabitants of the other main islands [10]. These populations were considered suitable for studying the effects of latitude, because of the latitude difference of at least $7^{\circ}$ between Hokkaido Island and southern Japan. In total, 247 patients with MS and 159 healthy controls (HCs) in the northern group, and 187 patients with MS and $235 \mathrm{HCs}$ in the southern group, participated in this study. Although sex and age were not matched between MS patients and HCs, HCs were used only for comparing HLA phenotypic frequency. Therefore, we believe that the use of unmatched HCs would not distort our results. We collected the demographic and clinical data of the participants including sex, age, age at disease onset, disease duration, MS subtype, annualized relapse rate (ARR), Kurtzke's Expanded Disability Status Scale (EDSS) [19] score, and Multiple Sclerosis Severity Score (MSSS) [20] 
at the time of DNA blood sampling. Fulfillment of the Barkhof criteria for MS [9], positivity of CSF OB (determined by isoelectric focusing) [21], and abnormality of IgG index as calculated by (CSF IgG/serum IgG)/(CSF albumin/serum albumin) were determined by retrospective review of each participant's medical records. In all patients, the presence of $\mathrm{OB}$ in CSF was assessed in a single laboratory (LSI Medience Corporation, Tokyo, Japan) and EDSS scores measured during remission were used. MSSS, which adjusts the EDSS score according to disease duration, was used to estimate the disease severity for each individual patient on a cross-sectional basis [20]. We defined the presence of Barkhof brain lesions by fulfillment of the four dichotomized MRI parameters proposed by Barkhof et al.: (1) the presence of at least one gadolinium-enhancing lesion or nine T2 hyperintense lesions, (2) the presence of at least one infratentorial lesion, (3) the presence of at least one juxtacortical lesion, and (4) the presence of at least three periventricular lesions [9]. We included cerebellar lesions as infratentorial lesions in the criteria. The IgG index was considered to be elevated if it was $>0.658$ [22]. We defined secondary progressive MS (SPMS) as a sustained increase in $\geq 1$ point when EDSS was $\leq 5.5$ and as a sustained increase in EDSS of $\geq 0.5$ point when EDSS was $\geq 6.0$ [23]. Benign MS was defined by an EDSS score of $\leq 2.0$ at least 10 years after disease onset [24]. Patients and HCs were recruited from June 2012 to January 2013 in the northern group and from April 2006 to January 2013 in the southern group [6]. As the prevalence of MS is lower in southern Japan than in northern Japan [25], a longer recruitment period was allowed in the southern group. The sample sizes required to reveal an association of the HLA-DRB1*04:05 allele with MS were calculated to be 122 cases and 122 controls, based on our previous report [6]. We also collected clinical data from 109 of the 187 southern patients up to January 2013 to adjust for the different recruitment periods between the groups. The ethics committees of each institution approved this study, and written informed consent was obtained from all participants.

\section{HLA-DRB1 and HLA-DPB1 genotyping}

The genotypes of the participants HLA-DRB1 and HLA$D P B 1$ alleles were determined by hybridization between polymerase chain reaction amplification products of the $H L A-D R B 1$ and $H L A-D P B 1$ genes and sequence-specific oligonucleotide probes, as previously described [26].

\section{Statistical analysis}

The phenotypic frequencies of the HLA-DRB1 and $H L A-D P B 1$ alleles were compared using either the chi-square test or Fisher's exact probability test (when criteria for the chi-square test were not fulfilled). The chi-square test was used to compare sex, SPMS, brain MRI lesions meeting the Barkhof criteria, and CSF IgG abnormalities between subgroups. We performed the Shapiro-Wilk test for normality for continuous variables, such as age, age at onset, disease duration, ARR, EDSS, and MSSS. As these continuous variables were not normally distributed, we used the MannWhitney $U$ test for comparisons between subgroups. The Mann-Whitney $U$ test was also used to compare MSSS between patients with and without Barkhof brain lesions and between patients with and without CSF IgG abnormalities.

A multivariate linear regression model was used for continuous dependent variables (log-transformed age at onset), while a logistic regression model was used for binary dependent variables (benign MS, fulfillment of Barkhof criteria for MS, and CSF IgG abnormalities), adjusting for other factors associated with dependent variables. We divided MSSS into quartiles defined by MSSS values of $0.94,2.60$, and 5.50, and a logistic regression model was used. All analyses were performed using JMP 11.0.0 (SAS Institute Inc., Cary, NC, USA). Statistical significance was set at $p<0.05$. Uncorrected $p$ values $\left(p^{\text {uncorr }}\right)$ were corrected by multiplying them by the number of comparisons (Bonferroni-Dunn's correction) to calculate corrected $p$ values $\left(p^{\text {corr }}\right)$.

\section{Results}

\section{Demographic features}

The clinical features of the 434 patients with MS (314 females, 120 males) are summarized in Table 1 . The median age at disease onset was 29 years (interquartile range $22-38$ years). The frequency of SPMS was $17.1 \%$. The median EDSS score was 2.0 (interquartile range 1.0-3.5), and the median MSSS was 2.60 (interquartile range $0.94-5.50)$. A total of 318 patients (74.3\%) had Barkhof brain lesions, and 193 patients (58.7 \%) had CSF IgG abnormalities.

\section{Frequencies of the HLA-DRB1 and HLA-DPB1 alleles}

As shown in Table 2, the phenotypic frequencies of the DRB1*04:05 and DRB1*15:01 alleles were significantly higher in MS patients than HCs $\left(p^{\text {corr }}=0.0004\right.$ and $p^{\text {corr }}=$ 0.0019 , respectively). The phenotypic frequencies of DRB1*01:01, DRB1*09:01, DRB1*13:02, and DRB1*15:02 were significantly lower in MS patients than $\mathrm{HCs}\left(p^{\text {corr }}=\right.$ $0.003, p^{\text {corr }}=0.0198, p^{\text {corr }}=0.0066$, and $p^{\text {corr }}=0.0396$, respectively). No DPB1 alleles were associated with MS in Japan (Additional file 2: Table S1). Compared with HCs, the phenotype frequency of DRB1"04:05 was significantly higher in patients with MS in both northern and southern regions ( $p^{\text {corr }}=0.0224$ and $p^{\text {corr }}=0.0384$, respectively) (Additional file 2: Tables S2 and S3). There was no 
Table 1 Demographic features of patients with MS according to region

\begin{tabular}{|c|c|c|c|c|}
\hline & Total patients $(n=434)$ & Northern patients $(n=247)$ & Southern patients $(n=187)$ & $p$ value \\
\hline Number of males/females (ratio) & 120/314 (1:2.6) & $62 / 185(1: 3.0)$ & $58 / 129(1: 2.2)$ & NS \\
\hline Age $(\text { years })^{a}$ & $41(33-50)$ & $41(34-50)$ & $40(32-50)$ & NS \\
\hline Age at onset (years) ${ }^{a}$ & $29(22-38)$ & $29(22-36)$ & $30(23-40)$ & NS \\
\hline SPMS (\%) & 73/427 (17.1) & $53 / 245(21.6)$ & 20/182 (11.0) & 0.0039 \\
\hline Disease duration (years) ${ }^{a}$ & $9(4-15)$ & $10(6-17)$ & $6.5(3-13)$ & $<0.0001$ \\
\hline $\mathrm{EDSS}^{\mathrm{a}}$ & $2.0(1.0-3.5)$ & $2.0(1.0-3.5)$ & $2.0(1.0-3.5)$ & NS \\
\hline $\mathrm{MSSS}^{\mathrm{a}}$ & $2.60(0.94-5.50)$ & $2.13(0.69-5.24)$ & $3.54(1.45-5.90)$ & 0.003 \\
\hline $\mathrm{ARR}^{\mathrm{a}}$ & $0.41(0.22-0.75)$ & $0.41(0.24-0.73)$ & $0.40(0.20-0.89)$ & NS \\
\hline Barkhof criteria (\%) & $318 / 428(74.3)$ & 198/247 (80.2) & $120 / 181(66.3)$ & 0.0012 \\
\hline Positive OB and/or increased lgG index (\%) & $193 / 329(58.7)$ & 130/177 (73.5) & $63 / 152(41.5)$ & $<0.0001$ \\
\hline Phenotypic frequency of HLA-DRB1*04:05 (\%) & $172(39.6)$ & $92(37.3)$ & $80(42.8)$ & NS \\
\hline Phenotypic frequency of HLA-DRB1*15:01 (\%) & $125(28.8)$ & $75(30.4)$ & $50(26.7)$ & NS \\
\hline
\end{tabular}

The Mann-Whitney $U$ test was used to compare continuous variables, and the chi-square test was used to compare categorical variables

$A R R$ annualized relapse rate, EDSS Kurtzke's Expanded Disability Status Scale, MSSS Multiple Sclerosis Severity Score, NS not significant, OB oligoclonal IgG bands,

SPMS secondary progressive multiple sclerosis

${ }^{a}$ Median (interquartile range)

significant difference in the distribution of $D R B 1$ alleles between northern and southern HCs (Additional file 2: Table S4).

\section{Comparison of clinical characteristics between northern and southern patients}

Northern MS patients had significantly lower MSSS scores compared with southern patients $(p=0.003)$ (Table 1), although the frequency of SPMS was higher in the north than in the south $(p=0.0039)$. Northern patients had a significantly longer disease duration, a higher frequency of Barkhof brain lesions, and a higher frequency of CSF IgG abnormalities compared with southern patients $(p<0.0001, p=0.0012$, and $p<0.0001$, respectively). Even after excluding all SPMS patients, northern patients had a significantly longer disease duration, lower EDSS and MSSS, and higher frequencies of Barkhof brain lesions and CSF IgG abnormalities compared with southern patients $(p=0.0004, p=0.0008, p<$ $0.0001, p=0.0217$, and $p<0.0001$, respectively) (Additional file 2: Table S5). Similar results were seen when comparing MS patients between northern and southern Japan. There were no significant differences in the phenotypic frequencies of $H L A-D R B 104: 05$ and $H L A-D R B 1$ 15:01 between northern and southern patients (37.3 vs. $42.8 \%, p=0.2432$; 30.4 vs. $26.7 \%, p=0.4087$, respectively) (Table 1). After excluding all SPMS patients, there were still no differences in the frequencies of either allele between northern and southern patients (HLA-DRB1 04:05, 37.6 vs. $43.7 \%, p=0.2403$; HLA-DRB1 15:01, 30.9 vs. $27.5 \%, p=0.4816$ ) (Additional file 2: Table S5).

When we compared the demographic features of northern and southern patients with using clinical data from southern patients that were followed up until
January 2013, there were no significant differences in disease duration, EDSS, or MSSS, although SPMS frequency was significantly higher in northern Japan ( $p=$ 0.0256) (Additional file 2: Table S6). When we excluded SPMS patients from the MS patient group, to adjust for the different recruitment periods, the northern patients had significantly lower MSSS (median 1.45 vs. 2.23, $p=$ 0.0411) (Additional file 2: Table S7).

We examined the association of MSSS with Barkhof brain lesions and CSF IgG abnormalities and found that neither was associated with MSSS in the total population nor in the northern or southern MS patient populations (Additional file 2: Table S8).

\section{Comparison of clinical characteristics between $H L A-D R B 1 * 04: 05$-positive and $H L A-D R B 1 * 04: 05$-negative patients}

MS patients with $D R B 1 * 04: 05$ were younger at disease onset, had a lower MSSS score, and had lower frequencies of Barkhof brain lesions and CSF IgG abnormalities than those without DRB1*04:05 ( $p=0.0003, p=0.0415, p=$ 0.0026 , and $p<0.0001$, respectively) (Table 3 ). In southern MS patients, $D R B 1 * 04: 05$ carriers were younger and had an earlier onset, longer disease duration, lower EDSS and MSSS scores, and lower frequencies of Barkhof brain lesions and CSF IgG abnormalities than non-DRB1*04:05 carriers $(p=0.0334, p=0.0001, p=0.0133, p=0.0263, p=$ $0.0021, p=0.0143$, and $p=0.008$, respectively). In northern patients, $D R B 1 \% 04: 05$ carriers also had a lower frequency of CSF IgG abnormalities than non-DRB1*04:05 carriers $(p=$ 0.0007), while age at onset, MSSS, and Barkhof brain lesion frequency in northern patients showed similar trends to the southern patients, but were not significantly different between DRB1*04:05 carriers and non-DRB1*04:05 carriers. 
Table 2 Phenotypic frequencies of HLA-DRB1 alleles in patients with MS

\begin{tabular}{|c|c|c|c|c|c|c|}
\hline \multirow[t]{2}{*}{$\overline{D R B 1}$} & \multicolumn{2}{|c|}{ Phenotype frequency, $n$ (\%) } & \multirow[t]{2}{*}{ OR } & \multirow[t]{2}{*}{$95 \% \mathrm{Cl}$} & \multirow[t]{2}{*}{$p^{\text {uncorr }}$} & \multirow[t]{2}{*}{$p^{\text {corr }}$} \\
\hline & MS $(n=434)$ & $\mathrm{HCS}(n=394)$ & & & & \\
\hline 01:01 & $24(5.5)$ & $53(13.5)$ & 0.38 & $0.23-0.62$ & $<0.0001$ & 0.003 \\
\hline 03:03 & $1(0.2)$ & 0 & NA & NA & 1.0000 & NS \\
\hline 04:01 & $7(1.6)$ & $9(2.3)$ & 0.70 & $0.26-1.90$ & 0.6151 & NS \\
\hline 04:03 & $42(9.7)$ & $22(5.6)$ & 1.81 & $1.06-3.09$ & 0.0276 & NS \\
\hline 04:04 & $2(0.5)$ & $2(0.5)$ & 0.91 & $0.13-6.47$ & 1.0000 & NS \\
\hline 04:05 & $172(39.6)$ & $100(25.4)$ & 1.93 & $1.43-2.60$ & $<0.0001$ & 0.0004 \\
\hline 04:06 & $42(9.7)$ & $26(6.6)$ & 1.52 & $0.91-2.52$ & 0.1071 & NS \\
\hline 04:07 & $2(0.5)$ & $4(1.0)$ & 0.45 & $0.08-2.48$ & 0.4317 & NS \\
\hline 04:10 & $24(5.5)$ & $8(2.0)$ & 2.82 & $1.25-6.36$ & 0.0091 & NS \\
\hline 07:01 & $1(0.2)$ & $2(0.5)$ & 0.45 & $0.04-5.01$ & 0.6073 & NS \\
\hline 07:10 & $1(0.2)$ & 0 & NA & NA & 1.0000 & NS \\
\hline 08:01 & $1(0.2)$ & $1(0.3)$ & 0.91 & $0.06-14.56$ & 1.0000 & NS \\
\hline 08:02 & $38(8.8)$ & $29(7.4)$ & 1.21 & $0.73-2.00$ & 0.4622 & NS \\
\hline 08:03 & $63(14.5)$ & $56(14.2)$ & 1.02 & $0.69-1.51$ & 0.9012 & NS \\
\hline 09:01 & $72(16.6)$ & $104(26.4)$ & 0.55 & $0.40-0.78$ & 0.0006 & 0.0198 \\
\hline 10:01 & $2(0.5)$ & $2(0.5)$ & 0.91 & $0.13-6.47$ & 1.0000 & NS \\
\hline 11:01 & $18(4.2)$ & $19(4.8)$ & 0.85 & $0.44-1.65$ & 0.6388 & NS \\
\hline $12: 01$ & $26(6.0)$ & $38(9.6)$ & 0.60 & $0.36-1.00$ & 0.0493 & NS \\
\hline 12:02 & $5(1.2)$ & $15(3.8)$ & 0.29 & $0.11-0.82$ & 0.0211 & NS \\
\hline 13:01 & $3(0.7)$ & $2(0.5)$ & 1.36 & $0.23-8.21$ & 1.0000 & NS \\
\hline 13:02 & $20(4.6)$ & $46(11.7)$ & 0.37 & $0.21-0.63$ & 0.0002 & 0.0066 \\
\hline 13:07 & 0 & $1(0.3)$ & 0 & NA & 0.4758 & NS \\
\hline 14:01 & $3(0.7)$ & 0 & NA & NA & 0.2508 & NS \\
\hline 14:02 & $1(0.2)$ & $1(0.3)$ & 0.91 & $0.06-14.56$ & 1.0000 & NS \\
\hline 14:03 & $20(4.6)$ & $14(3.6)$ & 1.31 & $0.65-2.63$ & 0.4449 & NS \\
\hline 14:05 & $17(3.9)$ & $15(3.8)$ & 1.03 & $0.51-2.09$ & 0.9347 & NS \\
\hline 14:06 & $11(2.5)$ & $6(1.5)$ & 1.68 & $1.62-4.59$ & 0.3371 & NS \\
\hline 14:07 & 0 & $2(0.5)$ & 0 & NA & 0.2261 & NS \\
\hline $14: 54$ & $20(4.6)$ & $28(7.1)$ & 0.63 & $0.35-1.14$ & 0.1245 & NS \\
\hline 15:01 & $125(28.8)$ & $67(17.0)$ & 1.97 & $1.41-2.76$ & $<0.0001$ & 0.0019 \\
\hline 15:02 & 55 (12.7) & 83 (21.1) & 0.54 & $0.37-0.79$ & 0.0012 & 0.0396 \\
\hline $15: 10$ & $1(0.2)$ & 0 & NA & NA & 1.0000 & NS \\
\hline 16:02 & $4(0.9)$ & $4(1.0)$ & 0.91 & $0.23-3.65$ & 1.0000 & NS \\
\hline
\end{tabular}

$p^{\text {uncorr }}$ was corrected by multiplying the value by 33 to calculate $p^{\text {corr }}$

$\mathrm{Cl}$ confidence interval, HCs healthy controls, MS multiple sclerosis, NA not applicable, NS not significant, OR odds ratio, $p^{\text {corr }}$ corrected $p$ value

\section{Comparison of clinical characteristics between HLA-DRB 1*15:01-positive and HLA-DRB1*15:01-negative patients}

$H L A-D R B 1 * 15: 01$ carriers had a significantly higher frequency of CSF IgG abnormalities than non-DRB1*15:01 carriers $(p=0.0058$, Additional file 2: Table S9). The effect of $D R B 1 * 15: 01$ on CSF IgG abnormalities was significant in southern patients, but not in northern patients $(p=0.0002$ and $p=0.9042$, respectively).
Comparison of clinical characteristics of $H L A-D R B 1 * 04: 05$ - or HLA-DRB 1*15:01-positive patients between northern and southern Japanese populations

We compared the clinical features of MS patients with the HLA-DRB1*04:05 or HLA-DRB1*15:01 allele between northern and southern Japan, respectively (Table 4 and Additional file 2: Table S10). MS patients with $D R B 1 * 04: 05$ in northern Japan showed higher frequencies of Barkhof brain lesions and positive CSF IgG 
Table 3 Comparison of the clinical features of patients with MS according to the presence or absence of HLA-DRB $1^{* 04: 05}$

\begin{tabular}{|c|c|c|c|c|c|c|c|c|c|}
\hline \multirow[t]{2}{*}{ DRB1 } & \multicolumn{3}{|l|}{ Total patients } & \multicolumn{3}{|l|}{ Northern patients } & \multicolumn{3}{|l|}{ Southern patients } \\
\hline & 04:05 (+) $(n=172)$ & 04:05 (-) $(n=262)$ & $p$ value & 04:05 (+) $(n=92)$ & $04: 05(-)(n=155)$ & $p$ value & 04:05 (+) $(n=80)$ & 04:05 (-) $(n=107)$ & $p$ value \\
\hline Number of males/females (ratio) & $52 / 120(1: 2.3)$ & $68 / 194(1: 2.9)$ & NS & $27 / 65(1: 2.4)$ & $35 / 120(1: 3.4)$ & NS & $25 / 55(1: 2.2)$ & $33 / 74(1: 2.2)$ & NS \\
\hline Age $(\text { years })^{a}$ & $39(31-49)$ & $42(34.2-51.5)$ & 0.0259 & $41(34-49.5)$ & $42(35-51)$ & NS & $36(28-49)$ & $42(34-52)$ & 0.0334 \\
\hline Age at onset (years) ${ }^{a}$ & $26(21-34.8)$ & $30.2(24-40)$ & 0.0003 & $27(21-34)$ & $29(24-37)$ & NS & $25(20-36.8)$ & $33(26-43)$ & 0.0001 \\
\hline SPMS (\%) & $26 / 170(15.3)$ & $47 / 257(18.3)$ & NS & 19/92 (20.7) & $34 / 153(22.2)$ & NS & $7 / 78(9.0)$ & 13/104 (12.5) & NS \\
\hline Disease duration (years) ${ }^{a}$ & $9(5.3-16)$ & $9(4-14)$ & NS & $10(6-17)$ & $11(5-17)$ & NS & $7.5(4-14.8)$ & $5.5(3-10.3)$ & 0.0133 \\
\hline $\mathrm{EDSS}^{\mathrm{a}}$ & $2.0(1.0-3.5)$ & $2.0(1.0-3.5)$ & NS & $2.0(1.0-3.5)$ & $2.0(1.0-3.5)$ & NS & $2.0(1.0-3.0)$ & $2.5(1.5-4.0)$ & 0.0263 \\
\hline $\mathrm{MSSS}^{\mathrm{a}}$ & $2.10(0.78-5.24)$ & $3.17(1.16-5.87)$ & 0.0415 & $1.97(0.56-5.63)$ & $2.33(0.88-4.94)$ & NS & $2.28(1.04-4.97)$ & $4.32(1.77-6.33)$ & 0.0021 \\
\hline $\mathrm{ARR}^{\mathrm{a}}$ & $0.40(0.22-0.71)$ & $0.43(0.22-0.85)$ & NS & $0.40(0.25-0.71)$ & $0.42(0.22-0.75)$ & NS & $0.37(0.17-0.71)$ & $0.48(0.21-0.95)$ & NS \\
\hline Barkhof criteria (\%) & $113 / 170(66.5)$ & $205 / 258(79.5)$ & 0.0026 & 69/92 (75.0) & 129/155 (83.2) & NS & $44 / 78(56.4)$ & 76/103 (73.8) & 0.0143 \\
\hline $\begin{array}{l}\text { Positive OB and/or } \\
\text { increased lgG } \\
\text { index (\%) }\end{array}$ & $58 / 129(45.0)$ & $135 / 200(67.5)$ & $<0.0001$ & $41 / 69(59.4)$ & $89 / 108$ (82.4) & 0.0007 & $17 / 60(28.3)$ & $46 / 92(50.0)$ & 0.008 \\
\hline
\end{tabular}

The Mann-Whitney $U$ test was used to compare continuous variables, and the chi-square test was used to compare categorical variables

ARR annualized relapse rate, EDSS Kurtzke's Expanded Disability Status Scale, MSSS Multiple Sclerosis Severity Score, NS not significant, OB oligoclonal IgG bands, SPMS secondary progressive multiple sclerosis ${ }^{\mathrm{a}}$ Median (interquartile range) 
Table 4 Clinical characteristics of patients with MS and the HLA-DRB 1*04:05 allele

\begin{tabular}{|c|c|c|c|}
\hline & $\begin{array}{l}\text { Northern } \\
\text { patients } \\
(n=92)\end{array}$ & $\begin{array}{l}\text { Southern } \\
\text { patients } \\
(n=80)\end{array}$ & $p$ value \\
\hline $\begin{array}{l}\text { Number of males/females } \\
\text { (ratio) }\end{array}$ & $27 / 65(1: 2.4)$ & $25 / 55(1: 2.2)$ & NS \\
\hline Age (years) ${ }^{a}$ & $41(34-49.5)$ & $36(28-49)$ & NS \\
\hline Age at onset (years) ${ }^{a}$ & $27(21-34)$ & $25(20-36.8)$ & NS \\
\hline SPMS (\%) & 19/92 (20.7) & 7/78 (9.0) & 0.035 \\
\hline Disease duration (years) ${ }^{a}$ & $10(6-17)$ & $7.5(4-14.8)$ & NS \\
\hline EDSS $^{a}$ & $2.0(1.0-3.5)$ & $2.0(1.0-3.0)$ & NS \\
\hline MSSS $^{\mathrm{a}}$ & $1.97(0.56-5.63)$ & $2.28(1.04-4.97)$ & NS \\
\hline$A R R^{a}$ & $0.40(0.25-0.71)$ & $0.37(0.17-0.71)$ & NS \\
\hline Barkhof criteria (\%) & 69/92 (75.0 \%) & 44/78 (56.4 \%) & 0.0105 \\
\hline $\begin{array}{l}\text { Positive OB and/or } \\
\text { increased lgG index (\%) }\end{array}$ & 41/69 (59.4 \%) & 17/60 (28.3 \%) & 0.0004 \\
\hline
\end{tabular}

The Mann-Whitney $U$ test was used to compare continuous variables, and the chi-square test was used to compare categorical variables

$A R R$ annualized relapse rate, EDSS Kurtzke's Expanded Disability Status Scale,

MSSS Multiple Sclerosis Severity Score, OB oligoclonal lgG bands, SPMS

secondary progressive multiple sclerosis

${ }^{a}$ Median (interquartile range)

abnormalities compared with those in southern Japan $(p=0.0105$ and $p=0.0004)$. This suggests that higher latitude increases the frequencies of Barkhof brain lesions and CSF IgG abnormalities independent of the HLA-DRB1*04:05 allele. There were no significant differences between northern and southern Japan in the clinical features of MS patients with the DRB1*15:01 allele.

Multivariate regression analyses of factors contributing to clinical features and laboratory findings

We adjusted for factors contributing to age at onset, namely geographic region (northern vs. southern), sex, HLADRB1*04:05, and HLA-DRB1*15:01. Only the DRB1*04:05 allele was significantly associated with an earlier age at disease onset $(p<0.0001)$ (Table 5). After adjusting for factors contributing to MSSS, namely geographic region (northern vs. southern), sex, age at onset, MS subtype, ARR, HLA$D R B 1 * 04: 05$, and $H L A-D R B 1 * 15: 01$, patients living at a higher latitude and with the $H L A-D R B 1 * 04: 05$ allele were independently associated with the lowest quartile of MSSS (adjusted $\mathrm{OR}=3.64, p<0.0001$ and adjusted $\mathrm{OR}=1.81$, $p=0.0266$, respectively). Older age at onset, SPMS, and higher ARR were significantly associated with higher MSSS $(p=0.0159, p<0.0001$, and $p<0.0001$, respectively). When the logistic regression model for benign MS was used to adjust for the same confounding factors as MSSS, living at a higher latitude and carrying the HLA-DRB1*04:05 allele were significantly associated with benign MS (adjusted OR $=2.63, p=0.0206$; adjusted $\mathrm{OR}=2.49, p=0.0275$, respectively), while SPMS had a
Table 5 Multivariate analysis of factors contributing to clinical and laboratory features

\begin{tabular}{|c|c|c|c|}
\hline Age at disease onset & $\beta$ & $t$ value & $p$ value \\
\hline Region (northern Japan) & -0.0918 & -1.94 & NS \\
\hline Sex (female) & -0.0127 & -0.27 & NS \\
\hline HLA-DRB1*04:05 allele (+) & -0.1970 & -4.12 & $<0.0001$ \\
\hline HLA-DRB1*15:01 allele $(+)$ & -0.0239 & -0.50 & NS \\
\hline MSSS (Q1/Q2-Q4) & Adjusted OR & $95 \% \mathrm{Cl}$ & $p$ value \\
\hline Region (northern Japan) & 3.64 & $2.15-6.33$ & $<0.0001$ \\
\hline Sex (female) & 0.98 & $0.55-1.76$ & NS \\
\hline Age at onset & 0.97 & $0.95-0.99$ & 0.0159 \\
\hline SPMS & 0.06 & $0.009-0.20$ & $<0.0001$ \\
\hline ARR & 0.15 & $0.07-0.31$ & $<0.0001$ \\
\hline HLA-DRB $1^{*} 04: 05$ allele $(+)$ & 1.81 & $1.07-3.07$ & 0.0266 \\
\hline HLA-DRB1*15:01 allele $(+)$ & 1.46 & $0.83-2.56$ & NS \\
\hline Benign MS & Adjusted OR & $95 \% \mathrm{Cl}$ & $p$ value \\
\hline Region (northern Japan) & 2.63 & $1.18-6.09$ & 0.0206 \\
\hline Sex (female) & 0.87 & $0.37-2.00$ & NS \\
\hline Age at onset & 0.97 & $0.94-1.01$ & NS \\
\hline SPMS & 0.04 & $0.008-0.12$ & $<0.0001$ \\
\hline ARR & 0.43 & $0.11-1.42$ & NS \\
\hline HLA-DRB $1 * 04: 05$ allele $(+)$ & 2.49 & $1.12-5.74$ & 0.0275 \\
\hline HLA-DRB1*15:01 allele $(+)$ & 1.72 & $0.76-4.04$ & NS \\
\hline Barkhof criteria & Adjusted OR & $95 \% \mathrm{Cl}$ & $p$ value \\
\hline Region (northern Japan) & 1.63 & $1.01-2.63$ & 0.0434 \\
\hline Sex (female) & 0.80 & $0.46-1.35$ & NS \\
\hline Age at onset & 0.97 & $0.95-0.99$ & 0.0132 \\
\hline SPMS & 2.55 & $1.05-6.80$ & 0.0384 \\
\hline Disease duration (years) & 0.98 & $0.95-1.01$ & NS \\
\hline ARR & 1.05 & $0.67-1.74$ & NS \\
\hline EDSS & 0.98 & $0.85-1.13$ & NS \\
\hline HLA-DRB1 ${ }^{*} 04: 05$ allele $(+)$ & 0.49 & $0.30-0.80$ & 0.0043 \\
\hline HLA-DRB1*15:01 allele $(+)$ & 1.45 & $0.85-2.53$ & NS \\
\hline $\begin{array}{l}\text { Positive } O B \text { and/or increased } \\
\text { lgG index }\end{array}$ & Adjusted OR & $95 \% \mathrm{Cl}$ & $p$ value \\
\hline Region (northern Japan) & 3.80 & $2.31-6.36$ & $<0.0001$ \\
\hline Sex (female) & 1.36 & $0.78-2.36$ & NS \\
\hline Age at onset & 1.00 & $0.98-1.02$ & NS \\
\hline SPMS & 1.43 & $0.60-3.51$ & NS \\
\hline ARR & 0.99 & $0.66-1.49$ & NS \\
\hline EDSS & 1.00 & $0.87-1.16$ & NS \\
\hline HLA-DRB 1*04:05 allele $(+)$ & 0.39 & $0.23-0.65$ & 0.0004 \\
\hline HLA-DRB1*15:01 allele $(+)$ & 1.65 & $0.95-2.88$ & NS \\
\hline
\end{tabular}

Benign MS was defined as an EDSS score $\leq 2.0$ at least 10 years after disease onset $A R R$ annualized relapse rate, $C l$ confidence interval, EDSS Kurtzke's Expanded Disability Status Scale, MSSS Multiple Sclerosis Severity Score, NS not significant, $O B$ oligoclonal IgG bands, $O R$ odds ratio, $Q$ quartile, SPMS secondary progressive multiple sclerosis 
negative association $(p<0.0001)$. Results from the multivariate regression analyses for MSSS and benign MS were consistent with these results. Interestingly, MSSS was estimated to decrease by 0.57 per DRB $1 * 04: 05$ allele $(p$ $=0.0198$, Fig. 1), suggesting a gene-dosage effect. A younger age at onset also had a significant positive linear trend with dosage of $D R B 1 * 04: 05$ allele $(p=0.0027)$. After adjusting for the geographic region (northern vs. southern), sex, age at onset, MS subtype, disease duration, ARR, EDSS, HLA-DRB1:04:05, and HLA$D R B 1 * 15: 01$, we observed a positive correlation between living at a higher latitude and the presence of Barkhof brain lesions (adjusted $\mathrm{OR}=1.63, p=0.0434$ ) and a negative correlation between $H L-D R B 1 * 04: 05$ and Barkhof brain lesions (adjusted $\mathrm{OR}=0.49, p=0.0043$ ). Additionally, a younger age at onset and SPMS were positively associated with Barkhof brain lesions $(p=0.0132$ and $p=0.0384$, respectively). Living at a higher latitude also had a strong positive association with positive $\mathrm{OB}$ and/or increased IgG index (adjusted $\mathrm{OR}=3.80, p<0.0001$ ). $D R B 1 * 04: 05$ had a strong negative association with CSF IgG abnormalities (and adjusted OR $=0.39, p=0.0004$, respectively) after adjusting for geographic region (northern to southern), sex, age at onset, MS subtype, ARR, EDSS, HLA-DRB1*04:05, and HLA-DRB1*15:01.

\section{Discussion}

Using multivariate regression analyses, we found that living at a higher latitude and carrying the DRB1*04:05 allele independently lessened the severity of symptoms as evaluated by MSSS. The analyses also revealed that latitude significantly increases the emergence of two hallmarks of MS: fulfillment of the Barkhof criteria for MS, and CSF IgG abnormalities, and that DRB1*04:05 decreases the occurrence of these parameters. The

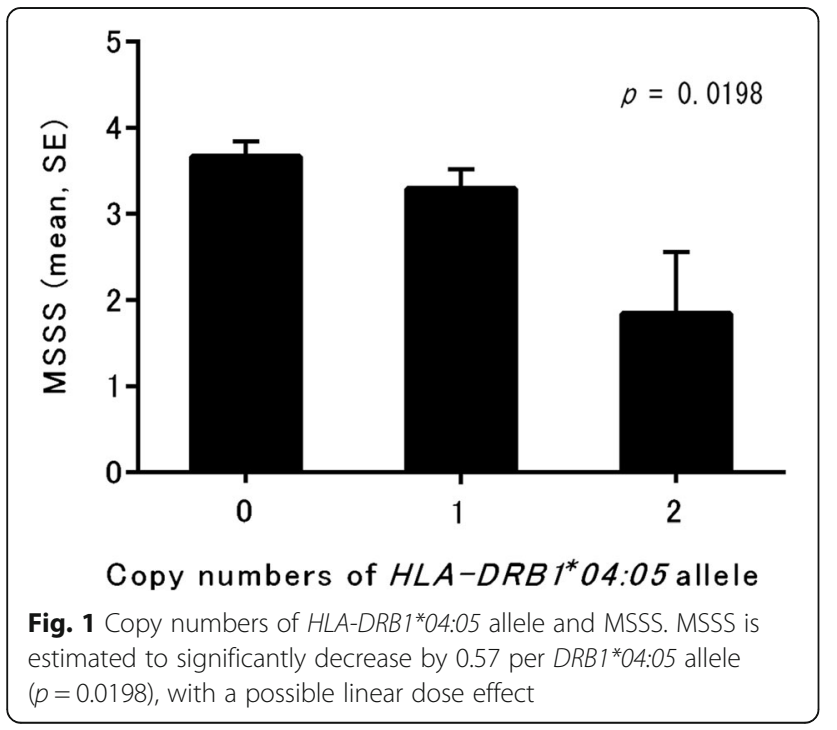

difference in clinical features between northern and southern patients was not simply attributable to the difference in the proportion of SPMS patients enrolled, as even after excluding these patients, the difference remained statistically significant.

Thus, DRB1*04:05 appears to decrease MSSS, the frequency of brain lesions meeting the Barkhof criteria, and CSF IgG abnormalities. This is in accordance with our previous report describing $D R B 1 * 04: 05$-positive MS to be a mild disease with lower frequencies of Barkhof brain lesions and CSF abnormalities [6]. DRB1*04 is also associated with OB-negative MS in a Swedish population [27]. Worldwide, the DRB1*04:05 allele is rare in the general population, but it is one of the most common alleles in some select and isolated populations, such as the Japanese, Sardinians, and Papua New Guineans [28]. DRB1:04:05 is a common susceptibility allele for MS in the Japanese population, and DR4 (DRB1*04:05-DQA1*05:01-DQB1*03:01) is also a susceptibility haplotype for MS in the Sardinian population [29], both of which recently showed a steep rise in MS prevalence [10,30]. We reported that the proportion of $D R B 1$ "04:05 carriers in MS patients rapidly increases with descending year of birth [6]. Thus, DRB1*04:05 is a potential risk factor for MS in the younger generation, which is growing up in a modernized environment. Surprisingly, in our study, DRB1*04:05 had a gene-dosage effect on MSSS, decreasing by 0.57 per DRB1*04:05 allele. Therefore, $D R B 1 * 04: 05$ appears to be unique in its ability to both increase susceptibility to MS and decrease its severity as defined by MSSS. It would be of interest to test whether the bidirectional effects of DRB1*04:05 are observed in other populations, such as Sardinians, in which DRB1*04:05 is a common susceptibility allele.

A higher latitude is known to increase MS risk, and this is also true for the Japanese population [25]. It is notable that among Japanese, latitude also has a strong influence on clinical and laboratory manifestations, being positively associated with fulfillment of the Barkhof criteria for MS and CSF IgG abnormalities, but with less severity MS in patients living in northern Japan. In this study, we also found no significant difference in $D R B 1$ phenotypic frequency between northern and southern controls, suggesting that the observed differences were not likely due to the underlying genetic variation between northern and southern populations. It is interesting to note that latitude also exerts an influence on clinical manifestations in the Japanese population on the mainland with relatively homogeneous $H L A$ backgrounds [31]. In New Zealand, a weak inverse relationship between latitude and disease severity has also been observed [32]. Thus, environmental factors related to latitude are assumed to be more important for producing differences in clinical features than $H L A$ backgrounds.

The effects of latitude are bidirectional; it decreases disease severity as defined by MSSS while increasing the 
frequencies of Barkhof brain lesions and CSF IgG abnormalities. These bidirectional effects, in part, mask the effects of $D R B 1 * 04: 05$ on MSSS and fulfillment of Barkhof criteria for MS in northern MS patients. SPMS was associated with greater MSSS, but the frequency of SPMS was smaller in the southern MS population than that in the north. This suggests that the greater severity of MS in the south is not related to a chronic progressive phase. After excluding SPMS patients, greater MSSS scores in the southern group than in the northern became more evident in this study. Therefore, the greater MSSS scores in southern MS patients might be related to neuroinflammation during relapse, as well as poor recovery. Total brain T2weighted lesion load on MRI has little to no correlation with MS disability [33, 34]. However, gray matter atrophy and cortical lesion burdens are the most significant MRI variables correlating with MS disability $[35,36]$. In the future, the measurement of brain parenchymal volume and atrophy, as well as the assessment of cortical demyelination using double-inversion recovery MRI images, are required in our cohort to elucidate the factors influencing the differences in MS severity between the two areas. Although we carefully excluded patients with longitudinally extensive spinal cord lesions, spinal cord lesion load and spinal cord atrophy should also be measured in the future. It is important to clarify the effects of latitude on these parameters.

The clinical features of MS in Asians have been reported to be different to those in patients in Western countries. In the fourth nationwide survey in Japan, SPMS was observed in $15.2 \%$ of patients with conventional (classical)-type MS [10], suggesting that SPMS is less common in Japanese patients than in Western patients. It has repeatedly been reported that Japanese patients with MS have low rates of CSF IgG abnormality and Barkhof brain lesions [10, 21, 37]. Some studies have reported that the presence of $\mathrm{OB}$ is associated with a worse MS prognosis in Caucasians [38, 39], while others found no significant difference in prognosis between OB-positive and OB-negative MS patients, including a Japanese cohort $[27,37,40]$. We found that neither the presence of Barkhof brain lesions nor CSF abnormalities was associated with MSSS, suggesting that these factors may not be decisive prognostic factors for MS in Japanese populations. We suggest that living at higher latitude might independently exert stronger effects on MS severity than CSF IgG abnormalities. Indeed, this hypothesis is consistent with findings that higher latitude is associated with milder disability in MS patients in New Zealand [32]. Further research is needed to elucidate other factors related to the association of latitude with MS severity in Japanese populations.

In our study, the difference in disease severity in northern patients, as shown by differences in MSSS between $D R B 1 * 04: 05$ carriers and non-carriers, is not as prominent as in southern patients. In southern patients, lower latitude and an absence of $D R B 1 \% 04: 05$ increased the median MSSS to 4.33 in the $D R B 1 * 04: 05$ noncarriers. Conversely, in northern patients, higher latitude and the presence of $D R B 1 * 04: 05$ decreased MSSS to 1.97 in $D R B 1 * 04: 05$ carriers. Because the latter value is close to the lower limit, this lowering effect may not be obvious. Alternatively, the effects of latitude might be more potent in DRB1*04:05 non-carriers than in DRB1*04:05 carriers. Further studies are needed to investigate the interactions between the two factors in larger cohorts.

The $D R B 1 * 15: 01$ allele is a strong genetic risk factor for MS in Caucasians and has been associated with earlier age at onset [4] and OB positivity [41]. In the present study, $D R B 1 * 15: 01$ was associated with CSF IgG abnormalities and marginally correlated with fulfillment of the Barkhof criteria for MS. However, these associations lost significance after adjusting for contributing factors. Thus, the effects of $D R B 1 * 15: 01$ on clinical manifestations may be smaller in Asian populations than in European populations.

We also found that $D R B 1 * 01: 01, D R B 1 * 09: 01$, $D R B 1 * 13: 02$, and $D R B 1 * 15: 02$ were protective alleles in the Japanese MS population. Based on a meta-analysis, $D R B 1 * 09: 01$ has been reported to be a protective allele for MS in a Chinese population, and this allele is more common in Asians than in other ethnic groups [42]. $D R B 1 * 01: 01, D R B 1 * 13: 02$, and DRB1*15:02 have been determined to be novel protective alleles for MS in Japanese populations by the present study. Among the Basque, a Caucasian population living in northern Spain and southwestern France, the DRB1*01:01 allele has a negative association with the disease [43]. The DRB1*15:02$D Q B 1 * 06: 01$ haplotype also decreases the risk for MS in a Sardinian population [44]. Interestingly, $D R B 1 * 15: 01$ and $D R B 1 * 15: 02$ only differ at amino acid position 86 (a valine for $D R B 1 * 15: 01$ and a glycine for $D R B 1 * 15: 02$ ). The difference of only one amino acid results in opposite effects on MS susceptibility. $D R B 1 * 15: 01$, which contains valine, only tolerates small residues, while $D R B 1 * 15: 02$, which contains glycine, can accommodate large aromatic peptide residues [45]. This small difference may determine the characteristic of presenting antigens, thereby resulting in opposite effects on MS.

Our study has some limitations; first, we did not measure the effects of disease-modifying drugs (DMDs) on clinical course. However, it would be difficult to explain the differences in the clinical manifestations and disease course according to regional and genetic factors based on DMD usage alone as such factors could not be taken into account in the use of DMDs in daily clinical practice. Second, we could not study the interactions between $H L A$ $D R B 1$ and environmental risk factors because of the small study sample size. The interaction between $D R B 1 * 15: 01$ and environmental risk factors, such as smoking, obesity, 
vitamin D, and Epstein-Barr virus infection, is well known [46-48]; any interaction between DRB1*04:05 and these environmental risk factors remains to be established. Third, although this is the largest study comparing clinical manifestations and genetic backgrounds between patients residing in northern and southern Japan, the sample size was still smaller than in Caucasian MS studies. Nevertheless, we believe it would be valuable to make further regional comparisons of the clinical manifestations and disease courses in genetically and culturally homogeneous populations residing in a wide range of latitudes to shed more light on the possible genetic and environmental influences on neuroinflammation.

\section{Conclusions}

Living at a higher latitude and carrying the DRB1*04:05 allele reduces the severity of MS in Japanese MS populations as defined by MSSS. However, both factors influence the frequencies of Barkhof brain lesions and CSF IgG abnormalities in different ways; that is, higher latitudes increase the frequencies of Barkhof brain lesions and CSF IgG abnormalities, whereas DRB1*04:05 decreases them. Further large-scale studies focusing on genetic-environmental interactions are needed to determine the factors associated with the severity of neuroinflammation in MS.

\section{Additional files}

Additional file 1: Figure S1. Map of Japanese main islands. The main islands of the Japanese archipelago span $31-46^{\circ}$ north [10]. The residing areas of northern MS patients recruited from the Hokkaido Island are located $42-45^{\circ}$ north, while those of southern MS patients recruited from the southern half of the Japanese archipelago span $33-35^{\circ}$ north. Thus, there are at least $7^{\circ}$ of difference between the residing areas of the northern and southern patients in the present study. (TIF $121 \mathrm{~kb}$ )

Additional file 2: Table S1. Phenotypic frequencies of HLA-DPB1 alleles in patients with MS. Table S2. Phenotypic frequencies of HLA-DRB1 alleles in MS patients from northern Japan. Table S3. Phenotypic frequencies of HLA-DRB1 alleles in MS patients from southern Japan. Table S4. Phenotypic frequencies of HLA-DRB1 alleles in HCs. Table S5. Demographic features of patients with MS, excluding SPMS, according to region. Table S6.

Comparison of MS demographic features between northern and southern patients using clinical data from southern patients followed up until January 2013. Table S7. Comparison of MS demographic features between northern and southern patients using clinical data from southern patients followed up until January 2013, excluding SPMS. Table S8. Comparison of MSSS between MS patients with and without Barkhof brain lesions, and between those with and without CSF IgG abnormalities. Table S9. Comparison of clinical features in patients with MS according to the presence or absence of HLA-DRB1*15:01. Table S10. Clinical characteristics of MS patients with the HLA-DRB1*15:01 allele. (DOCX 54 kb)

\section{Acknowledgements}

We are grateful to all the participants for providing the blood samples. We are also grateful to the neurologists from JMSGC for providing the DNA samples and clinical information for this study. The JMSGC members are the following: Drs. Yuji Kawano (Site Investigator, Omuta Hospital), Katsuichi Miyamoto (Site Investigator, Kinki University), Susumu Kusunoki (Chairperson, Kinki University), Yuji Nakatsuji (Site Investigator, Osaka University), Hideki Mochizuki (Chairman, Osaka University), Kazuhide Ochi (Site Investigator, Hiroshima University), Masayasu Matsumoto (Chairman, Hiroshima University), Fumitaka Shimizu (Site
Investigator, Yamaguchi University), Takeshi Kanda (Chairman, Yamaguchi University), Hirofumi Ochi (Site Investigator, Ehime University), Tetsuro Miki (Chairman, Ehime University), Kazumasa Okada (Site Investigator, University of Occupational and Environmental Health), Sadatoshi Tsuji (Chairman, University of Occupational and Environmental Health), Masami Tanaka (Department Head, Utano National Hospital, National Hospital Organization), Masaaki Niino (Site Investigator, Hokkaido Medical Center, National Hospital Organization), Seiji Kikuchi (Director, Hokkaido Medical Center, National Hospital Organization), Toshiyuki Fukazawa (Director, Sapporo Neurology Clinic), Shin Hisahara (Site Investigator, Sapporo Medical University), Shun Shimohama (Chairman, Sapporo Medical University), Kazuto Yoshida (Deputy Director, Asahikawa Red Cross Hospital), and Hideki Houzen (Department Head, Obihiro Kosei General Hospital). We thank Dr Junji Kishimoto (Centre for Clinical and Translational Research, Kyushu University Hospital, Kyushu University) for his assistance with the statistical analyses.

\section{Funding}

This study was supported in part by a Health and Labour Sciences Research Grant on Intractable Diseases (H26-Nanchitou (Nan)-Ippan-074) from the Ministry of Health, Labour, and Welfare, Japan; by the "Practical Research Project for Rare/Intractable Diseases" from Japan Agency for Medical Research and Development, AMED, Japan; and by a Grant-in-Aid for Scientific Research A (MEXT KAKENHI Grant No. 16H02657), a Grant-in-Aid for Scientific Research C (No. 15K09341), a Grant-in-Aid for Exploratory Research (MEXT KAKENHI Grant No. 15K15341), a Grant-in-Aid for Scientific Research on Innovative Areas (No. 25117012), and a "Glial Assembly" Grant-in Aid for Scientific Research on Innovative Areas (JSPS KAKENHI Grant No. 25117001) from the Japan Society for the Promotion of Science, Japan. None of the funding bodies played any role in the study design, data collection and analysis, data interpretation, or writing the manuscript.

\section{Availability of data and materials}

The data will be shared on request based on the guidelines of Kyushu University Hospital Ethics Committee.

\section{Authors' contributions}

YN and TM jointly conceived the study, performed the statistical analyses, and wrote the paper. SSa, MN, TF, SY, SH, NI, SSh, MW, KY, HH, YM, RY, and SK participated in the procedure development and collated the data. JK conceived the study, wrote the paper, and supervised this work. All authors reviewed, amended, and agreed on the final version of the manuscript.

\section{Competing interests}

TM received a grant and payment from Bayer Schering Pharma and Takeda Pharmaceutical Company for the development of educational presentations and has also received speaker honoraria from Mitsubishi Tanabe Pharma, Bayer Schering Pharma, and Biogen. MN has received funding for travel and/or speaker honoraria from Biogen Idec, Bayer Schering Pharma, Mitsubishi, and Tanabe Pharma Corporation; is part of the scientific advisory board for Biogen Idec; and has received research support from Grants-in-Aid for Scientific Research from the Ministry of Health, Labour and Welfare of Japan. TF serves/ has served on scientific advisory boards for Bayer Pharma, Biogen Idec, Mitsubishi Tanabe Pharma Corporation, Takeda Pharmaceutical Company, and Novartis Pharma and has received funding for travel and speaker honoraria from Bayer Pharma, Biogen Idec, Mitsubishi Tanabe Pharma Corporation, and Novartis Pharma. SSh has received speaker honoraria from Novartis Pharma, Boehringer Ingelheim, Kyowa Hakko Kirin, Dainippon Sumitomo Pharma, Takeda Pharmaceutical Company, Daiichi Sankyo Pharma, Jansen Pharma, Eisai Pharma, Biogen Idec, and Mitsubishi Tanabe Pharma Corporation and has received research support from Grants-in-Aid for Scientific Research from the Ministry of Health, Labour and Welfare of Japan and the Smoking Research Foundation. $\mathrm{HH}$ serves on scientific advisory boards for Biogen Idec, Novartis Pharma, and Mitsubishi Tanabe Pharma Corporation. JK is a consultant for Biogen Idec Japan and Medical Review; has received honoraria from Bayer Healthcare, Mitsubishi Tanabe Pharma, Nobelpharma, Otsuka Pharmaceutical, and Medical Review; and is funded by a research grant for Nervous and Mental Disorders from the Ministry of Health, Labour and Welfare, Japan, and grants from the Japan Science and Technology Agency and the Ministry of Education, Culture, Sports, Science and Technology, Japan. These sponsors had no control over the interpretation, writing, or publication of this work. YN, SSa, SY, SH, NI, MW, KY, YM, RY, and SK have nothing to declare. 


\section{Consent for publication}

Not applicable.

\section{Ethics approval and consent to participate}

The study received ethical approval from the Kyushu University Hospital Ethics Committee (approval number: 575-03), and written informed consent was obtained from all participants.

\section{Author details \\ 'Department of Neurology, Neurological Institute, Graduate School of Yamanote 5-jo 7-chome, Nishi-ku, Sapporo 063-0005, Japan. ${ }^{3}$ Sapporo Hokkaido Medical Center, Yamanote 5-jo 7-chome, Nishi-ku, Sapporo 063-0005, Japan \\ Received: 13 January 2016 Accepted: 20 August 2016 Published online: 06 September 2016} Medical Sciences, Kyushu University, 3-1-1 Maidashi, Higashi-ku, Fukuoka 812-8582, Japan. ${ }^{2}$ Department of Clinical Research, Hokkaido Medical Center, Neurology Clinic, 21-2-1, Kita 21-jo Higashi, Higashi-ku, Sapporo 065-0021, Japan. ${ }^{4}$ Department of Neurology, School of Medicine, Sapporo Medical University, South 1 West 16, Chuo-ku, Sapporo 060-8543, Japan. ${ }^{5}$ Department of Neurology, Asahikawa Red Cross Hospital, 1-1-1, Akebono 1-jo, Asahikawa 070-8530, Japan. ${ }^{6}$ Department of Neurology, Obihiro Kosei General Hospital, 8-1, Nishi 6-jo Minami, Obihiro 080-0016, Japan. Department of Neurology,

\section{References}

1. Trapp BD, Peterson J, Ransohoff RM, Rudick R, Mörk S, Bö L. Axonal transection in the lesions of multiple sclerosis. N Engl J Med. 1998;338:278-85.

2. Ebers $\mathrm{G}$. Environmental factors and multiple sclerosis. Lancet Neurol. 2008;7:268-77. doi:10.1016/S1474-4422(08)70042-5.

3. Lincoln MR, Montpetit A, Cader MZ, Saarela J, Dyment DA, Tiislar M, et al. A predominant role for the HLA class II region in the association of the $\mathrm{MHC}$ region with multiple sclerosis. Nat Genet. 2005;37:1108-12.

4. Sawcer S, Hellenthal G, Pirinen M, Spencer CC, Patsopoulos NA, Moutsianas L, et al. Genetic risk and a primary role for cell-mediated immune mechanisms in multiple sclerosis. Nature. 2011;476:214-9.

5. Patsopoulos NA, Barcellos LF, Hintzen RQ, Schaefer C, van Duijn CM, Noble $J A$, et al. Fine-mapping the genetic association of the major histocompatibility complex in multiple sclerosis: HLA and non-HLA effects. PLoS Genet. 2013;9:e1003926.

6. Yoshimura S, Isobe N, Yonekawa T, Matsushita T, Masaki K, Sato S, et al. Genetic and infectious profiles of Japanese multiple sclerosis patients. PLoS One. 2012;7:e48592.

7. Simpson S, Blizzard L, Otahal P, Van der Mei I, Taylor B. Latitude is significantly associated with the prevalence of multiple sclerosis: a metaanalysis. J Neurol Neurosurg Psychiatry. 2011;82:1132-41.

8. Ascherio A, Munger KL. Environmental risk factors for multiple sclerosis. Part II: noninfectious factors. Ann Neurol. 2007;61:504-13.

9. Barkhof F, Filippi M, Miller DH, Scheltens P, Campi A, Polman CH, et al. Comparison of MRI criteria at first presentation to predict conversion to clinically definite multiple sclerosis. Brain. 1997:120:2059-69.

10. Osoegawa M, Kira J, Fukazawa T, Fujihara K, Kikuchi S, Matsui M, et al. Temporal changes and geographical differences in multiple sclerosis phenotypes in Japanese: nationwide survey results over 30 years. Mult Scler. 2009;15:159-73.

11. Dobson R, Ramagopalan S, Davis A, Giovannoni G. Cerebrospinal fluid oligoclonal bands in multiple sclerosis and clinically isolated syndromes: a meta-analysis of prevalence, prognosis and effect of latitude. J Neurol Neurosurg Psychiatry. 2013;84:909-14.

12. Lechner-Scott J, Spencer B, de Malmanche T, Attia J, Fitzgerald M, Trojano $M$, et al. The frequency of CSF oligoclonal banding in multiple sclerosis increases with latitude. Mult Scler. 2012;18:974-82.

13. Niino M, Sato S, Fukazawa T, Yoshimura S, Hisahara S, Matsushita T, et al. Latitude and HLA-DRB1 alleles independently affect the emergence of cerebrospinal fluid lgG abnormality in multiple sclerosis. Mult Scler. 2015;21: 1112-20.

14. Polman $\mathrm{CH}$, Reingold SC, Banwell B, Clanet M, Cohen JA, Filippi M, et al. Diagnostic criteria for multiple sclerosis: 2010 revisions to the McDonald criteria. Ann Neurol. 2011;69:292-302. doi:10.1002/ana.22366.
15. Wingerchuk DM, Lennon VA, Pittock SJ, Lucchinetti CF, Weinshenker BG. Revised diagnostic criteria for neuromyelitis optica. Neurology. 2006:66:1485-9.

16. Wingerchuk DM, Lennon VA, Lucchinetti CF, Pittock SJ, Weinshenker BG. The spectrum of neuromyelitis optica. Lancet Neurol. 2007;6:805-15.

17. Greer JM. The role of HLA in MS susceptibility and phenotype. Curr Top Behav Neurosci. 2015;26:1-27.

18. Weinshenker BG, Santrach P, Bissonet AS, McDonnell SK, Schaid D, Moore SB, et al. Major histocompatibility complex class II alleles and the course and outcome of MS: a population-based study. Neurology. 1998;51:742-7.

19. Kurtzke JF. Rating neurologic impairment in multiple sclerosis: an expanded disability status scale (EDSS). Neurology. 1983;33:1444-52.

20. Roxburgh RH, Seaman SR, Masterman T, Hensiek AE, Sawcer SJ, Vukusic S, et al. Multiple sclerosis severity score: using disability and disease duration to rate disease severity. Neurology. 2005;64:1144-51.

21. Nakashima I, Fujihara K, Sato S, Itoyama Y. Oligoclonal IgG bands in Japanese patients with multiple sclerosis. A comparative study between isoelectric focusing with lgG immunofixation and high-resolution agarose gel electrophoresis. J. Neuroimmunol. 2005;159:133-6.

22. Kira J, Kanai T, Nishimura Y, Yamasaki K, Matsushita S, Kawano Y, et al. Western versus Asian types of multiple sclerosis: immunogenetically and clinically distinct disorders. Ann Neurol. 1996:40:569-74.

23. Kappos $\mathrm{L}$. Placebo-controlled multicentre randomised trial of interferon $\beta-1 b$ in treatment of secondary progressive multiple sclerosis. Lancet. 1998;352:1491-7.

24. Pittock SJ, McClelland RL, Mayr WT, Jorgensen NW, Weinshenker BG, Noseworthy J, et al. Clinical implications of benign multiple sclerosis: a 20-year population-based follow-up study. Ann Neurol. 2004:56:303-6.

25. Kira J. Multiple sclerosis in the Japanese population. Lancet Neurol. 2003;2:117-27.

26. Matsushita T, Matsuoka T, Isobe N, Kawano Y, Minohara M, Shi N, et al. Association of the HLA-DPB1*0501 allele with anti-aquaporin-4 antibody positivity in Japanese patients with idiopathic central nervous system demyelinating disorders. Tissue Antigens. 2009;73:171-6.

27. Imrell K, Landtblom AM, Hillert J, Masterman T. Multiple sclerosis with and without CSF bands: clinically indistinguishable but immunogenetically distinct. Neurology. 2006;67:1062-4.

28. González-Galarza FF, Takeshita LYC, Santos EJM, Kempson F, Maia MHT, da Silva ALS, et al. Allele frequency net 2015 update: new features for HLA epitopes, KIR and disease and HLA adverse drug reaction associations. Nucleic Acids Res. 2015;43:D784-8.

29. Marrosu MG, Murru MR, Costa G, Murru R, Muntoni F, Cucca F. DRB1-DQA1DQB1 loci and multiple sclerosis predisposition in the Sardinian population. Hum Mol Genet. 1998:7:1235-7.

30. Pugliatti M, Sotgiu S, Solinas G, Castiglia P, Pirastru Ml, Murgia B, et al. Multiple sclerosis epidemiology in Sardinia: evidence for a true increasing risk. Acta Neurol Scand. 2001:103:20-6.

31. Nakaoka H, Mitsunaga S, Hosomichi K, Shyh-Yuh L, Sawamoto T, Fujiwara T, et al. Detection of ancestry informative HLA alleles confirms the admixed origins of Japanese population. PLoS One. 2013;8:e60793.

32. Alla S, Pearson JF, Taylor BV, Roxburgh R, Clarke G, Miller DH, et al. An investigation of the relationship between latitude and multiple sclerosis severity in New Zealand. Mult Scler. 2016;22:705-7.

33. Paty DW, Li DK. Interferon beta- $1 \mathrm{~b}$ is effective in relapsing-remitting multiple sclerosis. II. MRI analysis results of a multicenter, randomized, double-blind, placebo-controlled trial. UBC MS/MRI Study Group and the IFNB Multiple Sclerosis Study Group. Neurology. 1993;43:662-7.

34. Lycklama À, Nijeholt GJ, Van Walderveen MAA, Castelijns JA, Van Waesberghe JHTM, Polman C, Scheltens P, et al. Brain and spinal cord abnormalities in multiple sclerosis: correlation between MRI parameters, clinical subtypes and symptoms. Brain. 1998;121:687-97.

35. Calabrese M, De Stefano N, Atzori M, Bernardi V, Mattisi I, Barachino L, et al. Detection of cortical inflammatory lesions by double inversion recovery magnetic resonance imaging in patients with multiple sclerosis. Arch Neurol. 2007;64:1416-22.

36. Harrison DM, Roy S, Oh J, Izbudak I, Pham D, Courtney S, et al. Association of cortical lesion burden on 7-T magnetic resonance imaging with cognition and disability in multiple sclerosis. JAMA Neurol. 2015;72:1004-12. 
37. Kikuchi S, Fukazawa T, Niino M, Yabe I, Miyagishi R, Hamada T, et al. HLArelated subpopulations of MS in Japanese with and without oligoclonal IgG bands. Human leukocyte antigen. Neurology. 2003;60:647-51.

38. Avasarala JR, Cross AH, Trotter JL. Oligoclonal band number as a marker for prognosis in multiple sclerosis. Arch Neurol. 2001;58:2044-5.

39. Joseph FG, Hirst CL, Pickersgill TP, Ben-Shlomo Y, Robertson NP, Scolding NJ. CSF oligoclonal band status informs prognosis in multiple sclerosis: a case control study of 100 patients. J Neurol Neurosurg Psychiatry. 2009;80:292-6.

40 Siritho S, Freedman MS. The prognostic significance of cerebrospinal fluid in multiple sclerosis. J Neurol Sci. 2009;279:21-5.

41 Wu J-S, Qiu W, Castley A, James I, Joseph J, Christiansen FT, et al. Presence of CSF oligoclonal bands (OCB) is associated with the HLA-DRB1 genotype in a West Australian multiple sclerosis cohort. J Neurol Sci. 2010;288:63-7.

42 Qiu W, James I, Carroll WM, Mastaglia FL, Kermode AG. HLA-DR allele polymorphism and multiple sclerosis in Chinese populations: a metaanalysis. Mult Scler. 2011;17:382-8.

43 Fernández O, R-Antigüedad A, Pinto-Medel MJ, Mendibe MM, Acosta N, Oliver B, et al. HLA class II alleles in patients with multiple sclerosis in the Biscay province (Basque Country, Spain). J Neurol. 2009;256:1977-88.

44 Cocco E, Sardu C, Pieroni E, Valentini M, Murru R, Costa G, et al. HLA-DRB1DQB1 haplotypes confer susceptibility and resistance to multiple sclerosis in Sardinia. PLoS One. 2012;7:e33972.

45 Vogt A, Kropshofer H, Kalbacher H, Kalbus M, Rammensee HG, Coligan JE, et al. Ligand motifs of HLA-DRB5*0101 and DRB1*1501 molecules delineated from self-peptides. J Immunol. 1994;153:1665-73.

46 Goodin DS. The pathogenesis of multiple sclerosis. Clin Exp Neuroimmunol. 2015;6:2-22.

47 De Jager PL, Simon KC, Munger KL, Rioux JD, Hafler DA, Ascherio A. Integrating risk factors HLA-DRB1* 1501 and Epstein-Barr virus in multiple sclerosis. Neurology. 2008;70:1113-8.

48 Hedström AK, Sundqvist E, Bäärnhielm M, Nordin N, Hillert J, Kockum I, et al. Smoking and two human leukocyte antigen genes interact to increase the risk for multiple sclerosis. Brain. 2011:134:653-64.

\section{Submit your next manuscript to BioMed Central and we will help you at every step:}

- We accept pre-submission inquiries

- Our selector tool helps you to find the most relevant journal

- We provide round the clock customer support

- Convenient online submission

- Thorough peer review

- Inclusion in PubMed and all major indexing services

- Maximum visibility for your research

Submit your manuscript at www.biomedcentral.com/submit 